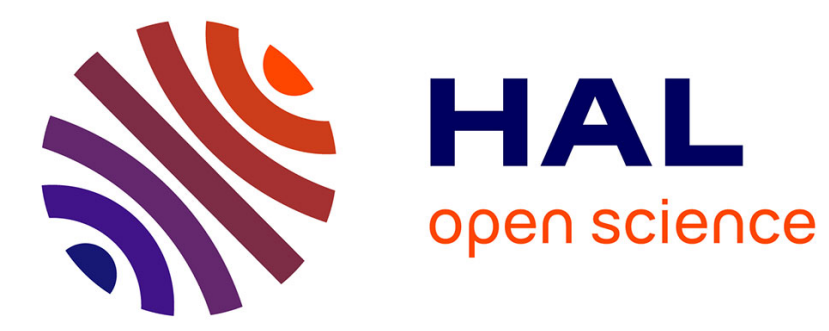

\title{
An adaptive pseudo-inverse method for the fault-tolerant output allocation in linear observers with redundant sensors
}

\author{
Andrea Cristofaro, Luca Zaccarian
}

\section{- To cite this version:}

Andrea Cristofaro, Luca Zaccarian. An adaptive pseudo-inverse method for the fault-tolerant output allocation in linear observers with redundant sensors. IEEE 55th Conference on Decision and Control (CDC 2016), Dec 2016, Las Vegas, United States. 6p., 10.1109/CDC.2016.7799098 . hal-01851154

\author{
HAL Id: hal-01851154 \\ https://hal.laas.fr/hal-01851154
}

Submitted on 12 Sep 2018

HAL is a multi-disciplinary open access archive for the deposit and dissemination of scientific research documents, whether they are published or not. The documents may come from teaching and research institutions in France or abroad, or from public or private research centers.
L'archive ouverte pluridisciplinaire HAL, est destinée au dépôt et à la diffusion de documents scientifiques de niveau recherche, publiés ou non, émanant des établissements d'enseignement et de recherche français ou étrangers, des laboratoires publics ou privés. 


\title{
An adaptive pseudo-inverse method for the fault-tolerant output allocation in linear observers with redundant sensors
}

\author{
Andrea Cristofaro and Luca Zaccarian
}

\begin{abstract}
We address the problem of output redundancy in linear plants, wherein the presence of redundant sensors is motivated by an unknown bias or fault affecting each sensor output. In this context, we address the design problem of a nonlinear observer consisting in a linear Luenberger structure augmented with an adaptive weighted pseudo-inverse combination of the available measurements. We characterize a number of properties of the proposed scheme and under certain conditions we establish local asymptotic stability of a certain attractor where the estimation error is zero and the fault is completely rejected. Simulation results are also given to show the potential behind the proposed solution.
\end{abstract}

\section{INTRODUCTION}

Input redundancy and control allocation has been widely investigated, to a large extent in the framework of control allocation [17], [10], [5], [19]. Conversely output allocation, or dual redundancy, is a less studied problem yet very interesting and many-sided [4]. The Kalman filter and the extended Kalman filter [11] [2] are estimation methods aiming at the minimization of the covariance error, and thus they benefit from the presence of a redundant set of outputs. However, such redundancy is usually not explicitly analyzed and is used in a passive way. As for the case of actuator redundancy, the presence of redundant outputs results in the potential enhancement of the estimation robustness to sensor faults and biased measurements. In this regard, some results pertaining safety and reliability of marine systems have been proposed [15]. On the other hand, sensor fault detection is a challenging problem, as there are no unquestionable methods to establish whether a sensor is faulty or not, based on the measurement value. Several approaches have been proposed [16], such as robust observers [3], [13], consensus based schemes [8], adaptive approximation [6], [18], [14] or statistical methods [12], [9]. Robust observers are typically designed for systems with structured faults by exploiting geometric properties to achieve fault decoupling. The underlying idea of consensus methods is to put more trust in the measurements on which the largest group of sensors agree, while adaptive approximation consists in the online reconstruction of a fault estimation, typically by means of neural networks architectures.

The method presented in this paper arises instead from the elementary observation that, among all the possible

A. Cristofaro is with Department of Engineering Cybernetics, Norwegian University of Science and Technology and with Center for Autonomous Marine Operations and Systems (AMOS), Trondheim, Norway. email: andrea.cristofaroditk.ntnu. no

L. Zaccarian is with LAAS-CNRS, Université de Toulouse, CNRS, Toulouse (France) and Dip. di Ingegneria Industriale, University of Trento (Italy).

This work is part of the work carried out at AMOS. It is supported by the Research Council of Norway through the Centres of Excellence funding scheme, project No. 223254 - AMOS, by the ANR project LimICoS contract number 12 BS03 005 01, by the iCODE institute, research project of the Idex Paris-Saclay, by grant OptHySYS funded by the University of Trento, and by grant PowerLyap funded by CaRiTRo. combinations of outputs, there is one which is less sensitive or even insensitive to a given fault. On the other hand, as the fault is typically unknown and unpredictable, such optimal combination is also generally unknown. In the absence of faults and external perturbations, an equivalent full-rank, lower dimensional output model can be extracted using a weighted pseudo-inverse, where the weights indicate the burden on each individual sensor. The introduction of dynamic pseudo-inverses allows to design a fault-tolerant adaptive observer with the nice property of being self-tuning. Moreover, the asymptotic value of the adaptive weights is a clear indication of faults presence, location and relative magnitude. One key-advantage of the proposed scheme is that it does not require any knowledge of the structure of the perturbations. Clearly, even though the approach has been developed with the aim of designing fault-tolerant observers, the setting is readily capable to handle different kinds of output perturbations, such as biased measurements [7] or outliers [1].

The paper is structured in four main sections. First in Section II-A the problem is formally stated, and the construction of the observers together with the dynamic pseudo-inverses is addressed in Section II-B, where the adaptation scheme is based on a modified gradient method. Some nice properties of the modified gradient operator are then exploited in Section IV and, under some assumptions on the system structure and the class of faults, local asymptotic stability is stated for the adaptive estimator composed by the observer and the dynamic weights. Finally, in Section V some numerical examples illustrate the method performances and support the theoretical results. All the proofs have been omitted from this conference publication due to space constraints.

\section{Proposed SCheme}

\section{A. Problem data}

Let us consider a linear plant

$$
\begin{aligned}
& \dot{x}=A x+B u \\
& y=C x+\phi
\end{aligned}
$$

where $x \in \mathbb{R}^{n}$ is the state, $u \in \mathbb{R}^{m}$ is the system input, and $y \in \mathbb{R}^{p}$ is the output. The unknown vector $\phi \in \mathbb{R}^{p}$ models faults or calibration errors possibly affecting the sensors.

We suppose that a redundant set of measurements is available, i.e.

$$
\operatorname{rank}(C)=q<p .
$$

The latter assumption may correspond to the presence of multiple identical sensors, as well as to the result of a suitable sensor fusion operation. Due to (2), matrices $H \in \mathbb{R}^{q \times n}$ and $Y \in \mathbb{R}^{p \times q}$ can be found such that

$$
C=Y H, \quad \operatorname{rank}(Y)=\operatorname{rank}(H)=q .
$$


As a matter of fact, an infinite number of such pairs $(Y, H)$ exist; having selected one, we refer to such $H$ as an extracted full rank (EFR) output matrix. In this regard, consider the virtual system

$$
\begin{aligned}
& \dot{x}_{v}=A x_{v}+B u \\
& z_{v}=H x_{v}
\end{aligned}
$$

where the EFR output matrix $H$ has been used. We need the following assumption.

Assumption 1: There exists a full-order observer for the virtual system (3), namely a system:

$$
\begin{aligned}
& \dot{\hat{x}}_{v}=A \hat{x}_{v}+B u+L\left(z_{v}-\hat{z}_{v}\right) \\
& \hat{z}_{v}=H \hat{x}_{v}
\end{aligned}
$$

satisfying for all initial conditions and all inputs $u$,

$$
\lim _{t \rightarrow \infty}\left|x_{v}(t)-\hat{x}_{v}(t)\right|=0 .
$$

Note that Assumption 1 implies that pair $(H, A)$ be detectable.

Definition 1: Given the redundant output matrix $C$, we denote by $\mathcal{C}_{\sharp}$ the set of matrix transformations that reduce $C$ to its EFR, i.e.

$$
\mathcal{C}_{\sharp}:=\left\{Z \in \mathbb{R}^{q \times p}: Z C=H\right\} .
$$

\section{B. Observer design}

Exploiting the output redundancy, the aim of this section is to design a self-tuning observer capable to select the best combination of outputs in order to optimize the state estimation in spite of the constant vector $\phi$ in (1).

Given the diagonal matrix $G_{\Gamma}=\operatorname{diag}(\Gamma)$ with $\Gamma=$ $\left[\begin{array}{lll}\gamma_{1} & \cdots & \gamma_{p}\end{array}\right]^{T}$, let us define the matrix

$$
Z_{\Gamma}=\left(Y^{T} G_{\Gamma} Y\right)^{-1} Y^{T} G_{\Gamma} .
$$

The latter is a weighted pseudo-inverse of $Y$ and, by construction, it verifies

$$
Z_{\Gamma} \in \mathcal{C}_{\sharp} \quad \forall \Gamma: \operatorname{det}\left(Y^{T} G_{\Gamma} Y\right) \neq 0 .
$$

Consider the following observer

$$
\begin{aligned}
& \dot{\hat{x}}=A \hat{x}+B u+L\left(Z_{\Gamma} y-\hat{z}\right) \\
& \hat{z}=H \hat{x},
\end{aligned}
$$

together with the adaptive parameter update equation

$$
\begin{aligned}
\dot{\Gamma}= & -\kappa_{\phi}\left|\operatorname{det}\left(Y^{T} G_{\Gamma} Y\right)\right|\left[\nabla_{\Gamma}\left|Z_{\Gamma} y-\hat{z}\right|^{2}\right]^{T} \\
& +\kappa_{\Gamma}\left(1-|\Gamma|^{2}\right) \Gamma
\end{aligned}
$$

where $\kappa_{\phi}, \kappa_{\Gamma}>0$ are rate parameters to be specified, and the operator $\nabla_{\Gamma}$ is the gradient with respect to $\left(\gamma_{1}, \ldots, \gamma_{p}\right)$.

Remark 1: We point out that the norm of $\Gamma$ is controlled by the second term in the right-hand side of (7), and therefore the vector of weights remains bounded. Moreover, in order to prevent an overflow in the computation of $Z_{\Gamma}$ the adaptation law might be enhanced with the introduction of a reset policy for reinitializing $\Gamma$ whenever $\operatorname{det}\left(Y^{T} G_{\Gamma} Y\right)$ becomes too close to zero. However, this goes beyond the scope of the present note, where we focus on a local analysis for which the condition $\operatorname{det}\left(Y^{T} G_{\Gamma} Y\right) \neq 0$ is uniformly guaranteed. 。

\section{AdAPTATION LAW AND STRUCTURAL PROPERTIES}

The adaptation law (7) consists of two terms. The first term is given by a gradient of the norm of the difference $Z_{\Gamma} y-\hat{z}$, rescaled by the determinant of $Y^{T} G_{\Gamma} Y$, and aims at minimizing the effect of the fault in the observer output injection (6); the second one, as already pointed out, is instead responsible of keeping the norm of $\Gamma$ unitary, this being compatible with the gradient-like optimization scheme thanks to the scale invariance of the pseudo-inverse $Z_{\Gamma}$.

It is useful to give an explicit expression of the gradient term in equation (7). In this perspective, the following technical result is needed.

Lemma 1: The gradient term appearing in (7) can be expressed as

$$
\begin{aligned}
& {\left[\nabla_{\Gamma}\left|Z_{\Gamma} y-\hat{z}\right|^{2}\right]^{T} } \\
= & 2 \operatorname{diag}\left(y-Y Z_{\Gamma} y\right) Y\left(Y^{T} G_{\Gamma} Y\right)^{-1}\left(Z_{\Gamma} y-\hat{z}\right)
\end{aligned}
$$

Using expression (8) it is straightforward to verify that, in nominal conditions, observer (6) guarantees the asymptotic estimation of state $x$, as formalized below.

Proposition 1: Let us consider system (1) without any output perturbation, that is $\phi \equiv 0$. Let the initial condition for the weight vector $\Gamma(0)$ be such that $Z_{\Gamma(0)} \in \mathcal{C}_{\sharp}$. Then the estimator (6)-(7) satisfies the asymptotic conditions

$$
\begin{aligned}
& \lim _{t \rightarrow \infty}|x(t)-\hat{x}(t)|=0, \\
& \lim _{t \rightarrow \infty} \Gamma(t)=\Gamma(0) /|\Gamma(0)| .
\end{aligned}
$$

The reason for introducing the adaptive scheme (7) is the attempt to design a sensor fault tolerant observer. For the sake of simplicity and in order to allow the statement of formal mathematical results, the fault $\phi$ is assumed to be constant, i.e. a step vector:

$$
\begin{array}{ll}
\phi(t)=0 & t<T_{0} \\
\phi(t)=\phi^{\star} & T \geq T_{0}
\end{array}
$$

where $T_{0}>0$ is the fault occurrence time. Consider the compact set:

$$
\mathcal{A}_{\Gamma}:=\left\{\Gamma \in \mathbb{R}^{p}: h_{\Gamma}:=Z_{\Gamma} \phi^{\star}=0 \text { and }|\Gamma|=1\right\},
$$

which is undetermined as long as the fault $\phi^{*}$ is unknown. It is easy to see that, whenever the vector $\Gamma$ of adaptive weights tends to $\mathcal{A}_{\Gamma}$, one has $Z_{\Gamma} y-\hat{z} \approx H(x-\hat{x})$ and hence the observer performances are recovered. The aim of this study is to investigate under what conditions the set $\mathcal{A}_{\Gamma}$ results to be attractive for state $\Gamma$ of the dynamical system (7) interconnected with the error dynamics of the proposed asymptotic observer. The following remark provides a first class of faults that must be excluded from the analysis.

Remark 2: Suppose that $\phi^{*} \in \operatorname{Im}(Y)$, in particular $\phi^{*}=$ $Y w$ for some $w \in \mathbb{R}^{q}$. Then one has

$$
\left(Y^{T} G_{\Gamma} Y\right)^{-1} Y^{T} G_{\Gamma} \phi^{*}=\left(Y^{T} G_{\Gamma} Y\right)^{-1} Y^{T} G_{\Gamma} Y w=w .
$$

As a consequence in this case the set $\mathcal{A}_{\Gamma}$ reduces to the empty set since one has $Z_{\Gamma} \phi^{*}=w$ for any admissible $\Gamma$. 。

The above remark shows that when $\phi^{*} \in \operatorname{Im}(Y)$, there is a complete loss of authority from $\Gamma$. This fact motivates the following assumption:

Assumption 2: The fault vector $\phi^{*}$ does not belong to the image of $Y$. 
We notice that nonsingularity of the matrix $Y^{T} G_{\Gamma} Y$ is a necessary property to be able to evaluate the gradient appearing in (7) and hence for the control architecture in (5)(7) to make sense. In this regard, we introduce an additional assumption based on the following family of sets.

Definition 2: For any $w \in \mathbb{R}^{q}$, we define the set $\mathcal{I}_{w}$ as

$$
\mathcal{I}_{w}=\operatorname{ker}\left(Y^{T} \operatorname{diag}(Y w)\right),
$$

and consider the class of admissible faults

$$
\Upsilon_{0}=\left\{\phi \in \mathbb{R}^{p}: \operatorname{ker}\left(Y^{T} \operatorname{diag}(\phi)\right) \cap \mathcal{I}_{w}=0 \forall w \in \mathbb{R}^{q}\right\} .
$$

Assumption 3: The fault vector $\phi^{*}$ verifies $\phi^{*} \in \Upsilon_{0}$.

Lemma 2: Under Assumption 2 and Assumption 3, for all $\Gamma^{*} \in \mathcal{A}_{\Gamma}$, matrix $Y^{T} G_{\Gamma^{*}} Y$ is nonsingular.

Remark 3: We notice that the set $\Upsilon_{0}$ is not a linear subspace, but a generalized cone with vertices in the origin. As a matter of fact, for any $\alpha \in \mathbb{R}$ and $\phi_{1}, \phi_{2} \in \Upsilon_{0}$ one has $\alpha \phi_{1} \in \Upsilon_{0}$ and $\alpha \phi_{2} \in \Upsilon_{0}$, while the inclusion $\phi_{1}+\phi_{2} \in \Upsilon_{0}$ in not guaranteed.

Remark 4: When $q=1$, the condition $\phi^{*} \in \Upsilon_{0}$ is always verified whenever $\phi^{*} \notin \operatorname{Im}(Y)$. When instead $q=p-1$ and $\phi^{*} \notin \operatorname{Im}(Y)$, the inclusion $\phi^{*} \in \Upsilon_{0}$ is enforced when $\operatorname{rank}\left(Y^{T} \operatorname{diag}\left(\phi^{*}\right)\right)=q$ and

$$
\operatorname{ker}\left(Y^{T} \operatorname{diag}(\phi)\right) \cap\left\{\Gamma: \Gamma_{j}>0 \forall j\right\} \neq 0 .
$$

\section{LOCAL ASYMPTOTIC STABILITY}

We are interested in the stability analysis for the dynamics of the estimation error $x-\hat{x}$, coupled with the adaptation law for $\Gamma$. In particular, in this section we will study the local asymptotic stability property of an attractor for the closedloop dynamics (6)-(7), wherein we have that the estimation error is zero and the state $\Gamma$ belongs to set $\mathcal{A}_{\Gamma}$ (this attractor is defined below in (14)).

We first exploit a helpful property, which allows to express the difference $Z_{\Gamma} y-\hat{z}$ in terms of the error $e$ and the steadystate fault value $\phi^{*}$. Observing that, for $t \geq T_{0}$, the output of the system verifies $y=C x+\phi^{*}=\bar{Y} H x+\phi^{*}$, it is straightforward to infer that

$$
\begin{aligned}
Z_{\Gamma} y-\hat{z} & =H x-H \hat{x}+Z_{\Gamma} \phi^{*} \\
& =H e+Z_{\Gamma} \phi^{*}=: H e+h_{\Gamma} .
\end{aligned}
$$

Moreover, the diagonal matrix in (8) satisfies the nice equivalence

$$
\begin{aligned}
\operatorname{diag}\left(y-Y Z_{\Gamma} y\right) & =\operatorname{diag}\left(Y H x+\phi^{*}-Y Z_{\Gamma}\left(Y H x+\phi^{*}\right)\right) \\
& =\operatorname{diag}\left(\phi^{*}-Y Z_{\Gamma} \phi^{*}\right),
\end{aligned}
$$

where the identity $Z_{\Gamma} Y=I$ has been used. The following property is useful to show that the dynamics of $h_{\Gamma}:=Z_{\Gamma} \phi^{*}$ is independent of the norm of $\Gamma$.

Lemma 3: Let $\Gamma$ be such that $\operatorname{det}\left(Y^{T} G_{\Gamma} Y\right) \neq 0$, and $\phi^{*} \in \mathbb{R}^{p}$. Then it holds

$$
\frac{\partial Z_{\Gamma} \phi^{*}}{\partial \Gamma} \Gamma=0
$$

To suitably characterize desirable properties of the observer (6)-(7), we define the estimation error as $e:=x-\hat{x}$ and compute the closed-loop error dynamics, resulting in:

$$
\begin{aligned}
\dot{e} & =(A-L H) e-L h_{\Gamma} \\
\dot{h}_{\Gamma} & =-2 \kappa_{\phi}\left|\operatorname{det}\left(Y^{T} G_{\Gamma} Y\right)\right| W^{T}(\Gamma) W(\Gamma)\left(H e+h_{\Gamma}\right),
\end{aligned}
$$

where we have used the equalities (also from Lemma 3):

$$
\dot{h}_{\Gamma}=\frac{\partial Z_{\Gamma} \phi^{*}}{\partial \Gamma} \dot{\Gamma}, \quad \kappa_{\Gamma}\left(1-|\Gamma|^{2}\right) \frac{\partial Z_{\Gamma} \phi^{*}}{\partial \Gamma} \Gamma=0,
$$

and we have set

$$
W(\Gamma):=\operatorname{diag}\left(\phi^{*}-Y h_{\Gamma}\right) Y\left(Y^{T} G_{\Gamma} Y\right)^{-1} .
$$

In light of the nonlinear error dynamics (13) we can now formally state the goal of this section, which is to establish conditions for local asymptotic stability of the following compact attractor:

$$
\mathcal{A}:=\left\{(e, \Gamma): e=0 \text { and } \Gamma \in \mathcal{A}_{\Gamma}\right\},
$$

where $\mathcal{A}_{\Gamma}$ has been defined in (12).

To establish some reasonable conditions for local asymptotic stability of $\mathcal{A}$, consider now any $\Gamma^{*} \in \mathcal{A}_{\Gamma}$. Then we get: $\operatorname{diag}\left(\phi^{*}-Y h_{\Gamma^{*}}\right)=\operatorname{diag}\left(\phi^{*}\right)$ from which we obtain

$$
\begin{aligned}
& W^{T}\left(\Gamma^{*}\right) W\left(\Gamma^{*}\right) \\
& \quad=\left(Y^{T} G_{\Gamma^{*}} Y\right)^{-1} Y^{T}\left[\operatorname{diag}\left(\phi^{*}\right)\right]^{2} Y\left(Y^{T} G_{\Gamma^{*}} Y\right)^{-1},
\end{aligned}
$$

where the two inverses exist from Lemma 2, as long as Assumptions 2 and 3 hold.

Motivated by the above equation and to simplify the stability analysis, we restrict the attention to the following class of faults:

Assumption 4: The fault vector $\phi^{*}$ satisfies

$$
\phi^{*} \in \Phi_{0}:=\left\{\varphi: \operatorname{det}\left(Y^{T}[\operatorname{diag}(\varphi)]^{2} Y\right) \neq 0\right\} .
$$

Restricting the attention to faults satisfying Assumption 4 is not a necessary assumption for exponential stability of $\mathcal{A}$ (for example, consider the case $\phi^{*}=0$ characterized in Proposition 1), but is a useful starting point to provide some conditions for the existence of strict Lyapunov functions. In particular, for each $\phi^{*} \in \Phi_{0}$, due to the fact that set $\mathcal{A}_{\Gamma}$ is compact and from the non-singularity condition established in Lemma 2, it is possible to find two positive scalars $\mu_{\min }$ and $\mu_{\max }$ such that for all $\Gamma \in \mathcal{A}_{\Gamma}$,

$$
\mu_{\min } I \leq \kappa_{\phi}\left|\operatorname{det}\left(Y^{T} G_{\Gamma} Y\right)\right| W^{T}(\Gamma) W(\Gamma) \leq \mu_{\max } I .
$$

This uniform positive definiteness property is especially useful if one considers a candidate Lyapunov function for the feedback interconnection (13) arising from some weighted sum of the two unperturbed systems, namely a Lyapunov function selected as:

$$
V\left(e, h_{\Gamma}\right):=\underbrace{e^{T} P e}_{:=V_{e}(e)}+\underbrace{\zeta_{1} h_{\Gamma}^{T} h_{\Gamma}}_{:=V_{\Gamma}(\Gamma)}+\zeta_{2}\left(1-|\Gamma|^{2}\right)^{2},
$$

where the two composing functions $V_{e}$ and $V_{\Gamma}$ are good Lyapunov functions for the non-interconnected system. More specifically, matrix $P$ can be selected as the solution to the following Lyapunov equation:

$$
(A-L H)^{T} P+P(A-L H)=-I,
$$

which always exists as long as Assumption 1 holds (note that more general choices may lead to less conservative stability conditions, but we regard this as future work with respect to this preliminary conference submission).

The arising local asymptotic stability result requires a specific inequality on the quantities defined above, as formalized in the next theorem. While this is a difficult condition to 
check in general, we provide next a corollary discussing a special case when this condition is automatically satisfied.

Theorem 1: Suppose that the steady-state fault value satisfies the inclusion $\phi^{*} \in \Phi_{0} \cap \Upsilon_{0}$ and $\phi^{*} \notin \operatorname{Im}(Y)$. If the following condition holds:

$$
4 \zeta_{1} \mu_{\min }>\left(|P L|+\zeta_{1}|H| \mu_{\max }\right)^{2} \text {, }
$$

then attractor (14) is locally asymptotically stable for the closed-loop error dynamics (13).

Corollary 1: If matrix $A$ is Hurwitz, then condition (19) always holds for a sufficiently small gain $L$ and a sufficiently small scalar $\zeta_{1}$. Therefore, attractor $\mathcal{A}$ is locally asymptotically stable.

Remark 5: The proposed technique also works reasonably well with time-varying faults $\phi(t)$ even though we do not establish formal results for this case. In particular, when $\phi$ is non-constant, parameters $\Gamma(t)$ aim at achieving some kind of output error attenuation. This generalization may be a key to enlarge the class of admissible faults. Indeed, the class $\Phi_{0}$ introduced in (15) may appear quite restrictive. However, from a practical point of view, the presence of noise in the measurements provides a persistence of excitation (PE) condition that can help the adaptation law to keep running even when the particular fault $\phi^{*}$ would make the matrix $W(\Gamma)$ become singular at certain time steps. Such a nice feature will be clearly illustrated in the simulation tests of Example 2, where a fault $\phi^{*}$ affecting a single sensor is considered, thus leading to $\operatorname{det}\left(Y^{T}\left[\operatorname{diag}\left(\phi^{*}\right)\right]^{2} Y\right)=0$ as long as matrix $Y$ has column dimension larger than 1 . In that case, fault rejection is obtained due the presence of additive noise on $\phi^{*}$.

\section{A simulation STUdy}

Let us consider a plant corresponding to (1) described by the following matrices:

$$
\begin{gathered}
A=\left[\begin{array}{rrr}
-1 & 3 & 0 \\
0.1 & -5 & 0.4 \\
0 & 0 & -5
\end{array}\right], \quad B=\left[\begin{array}{r}
1 \\
0 \\
-1
\end{array}\right] \\
C=Y H, \quad Y=\left[\begin{array}{ll}
1 & 1 \\
1 & 0 \\
2 & 1
\end{array}\right], \quad H=\left[\begin{array}{lll}
1 & 0 & 0 \\
0 & 1 & 0
\end{array}\right]
\end{gathered}
$$

Since matrix $A$ is Hurwitz, for the sake of simplicity and without loss of generality, no control input has been considered in the simulations, i.e. $u \equiv 0$. The same transient for the error dynamics would clearly be obtained for any nonzero selection of $u$. A bounded noise term affecting the outputs has been implemented in all the simulated scenarios to better illustrate the applicability of the result. For simplicity, in all our simulations, the gains $\kappa_{\phi}, \kappa_{\Gamma}$ have been chosen equal to the same value $\kappa_{\phi}=\kappa_{\Gamma}=\kappa$.

Example 1: Let us first assume that a sensor fault $\phi(t)$ occurs in the system according to the following scheme

$$
\phi(t)= \begin{cases}0 & t<60 \\ \phi^{*} & t \geq 60\end{cases}
$$

with $\phi^{*}=\left[\begin{array}{lll}3 & 0.5 & -1\end{array}\right]^{T}$. We select two different observer gains

$$
L_{1}=\left[\begin{array}{rr}
6 & 2.7 \\
-0.2 & -1 \\
-0.2 & -5.1
\end{array}\right], \quad L_{2}=\left[\begin{array}{rr}
8 & 2.9 \\
-0.1 & 8 \\
-1.4 & 37.5
\end{array}\right],
$$
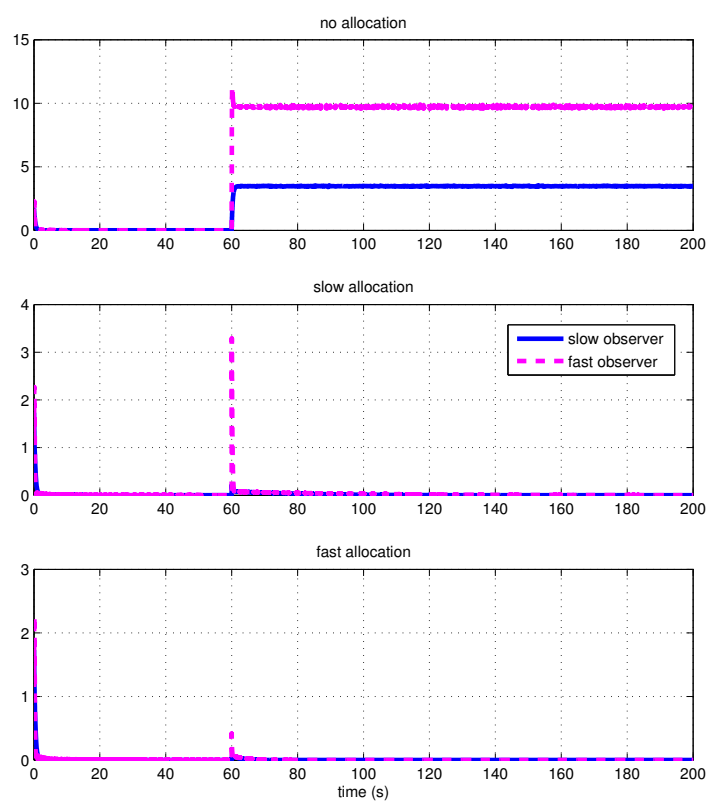

Fig. 1. Example 1: estimation error for three different cases: no adaptation, slow adaptation and fast adaptation.
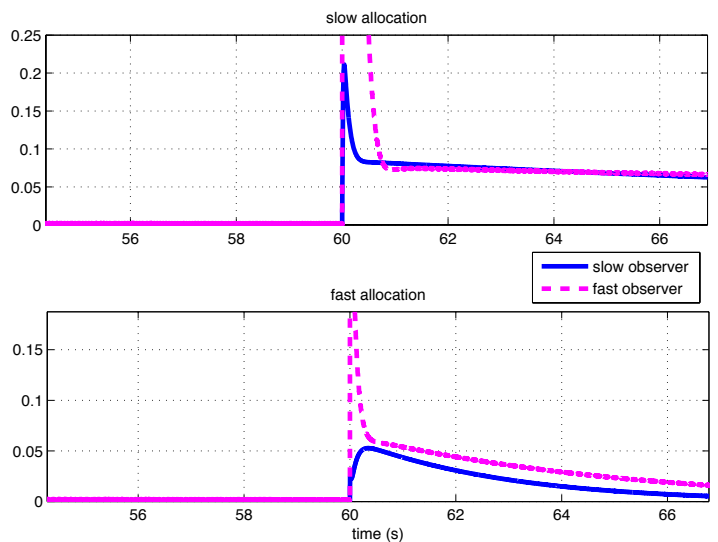

Fig. 2. Example 1: pseudo-inverse weights for slow and fast allocation.

corresponding to a slower and a faster estimator. Moreover, we have considered two different learning rates in the adaptation law, namely:

- slow allocation, $\kappa=\kappa_{\text {slow }}=1$,

- fast allocation, $\kappa=\kappa_{\text {fast }}=10$.

In both cases, the initial weight vector has been chosen as $\Gamma(0)=\frac{1}{\sqrt{3}}\left[\begin{array}{lll}1 & 1 & 1\end{array}\right]^{T}$, this being a natural choice corresponding to an equally distributed use of the sensors with $|\Gamma(0)|=1$.

The norms $|e|$ of the estimation errors in various scenarios are plotted in Figure 1. As expected, when no output allocation is implemented, namely when $\kappa=0$, the performance of the observers is corrupted by the fault effect: this is evident from the upper picture where the error $|e|$ remains nonzero both with the fast observer gain $L_{1}$ (magenta) and the slow observer gain $L_{2}$ (blue) in (20). Conversely, adjusting the pseudo-inverse weights according to the proposed adaptation law, the estimation of the system state is achieved notwith- 


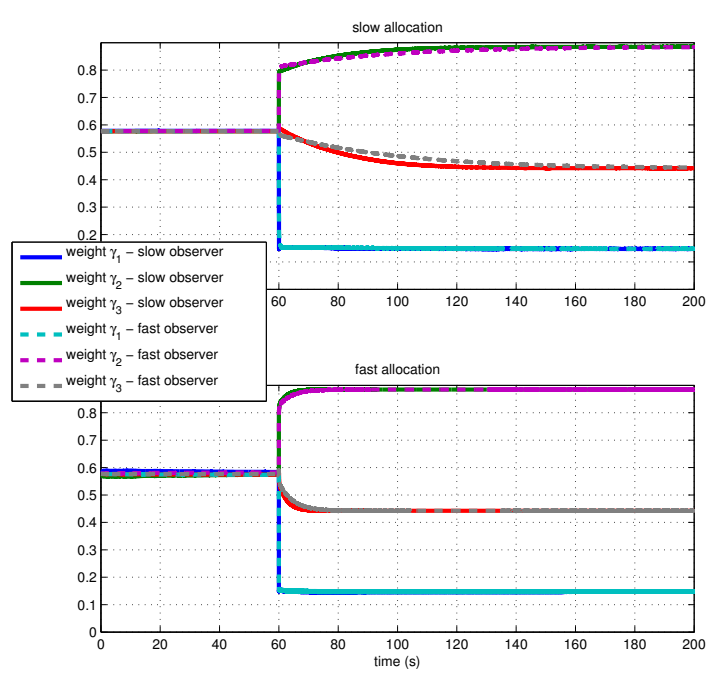

Fig. 3. Example 1: pseudo-inverse weights for slow and fast allocation.
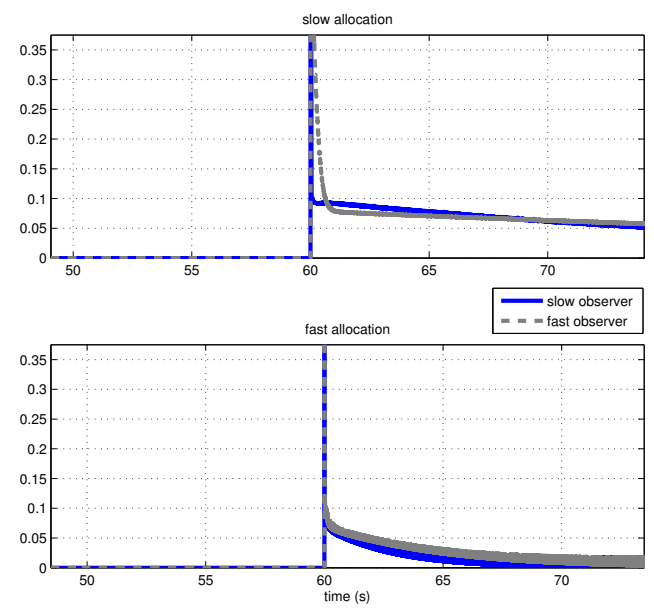

Fig. 4. Example 1: norm of the error signal $h_{\Gamma}$ (zoom).

standing the presence of the fault (see middle and bottom pictures). It is noticeable how the performances change as the parameters for the observer and the adaptation law vary. In particular, even though stability has been proved based on the assumption of $A$ being Hurwitz, it is interesting to note that the convergence rate depends indeed on the choice of the gain $L$ and not only on the open-loop properties of the plant. A zoom of the bottom traces of Figure 1 is plotted in Figure 2, in order to highlight the transient of the estimates. It is not surprising that the fast observer gain $L_{1}$ (magenta) injects a larger transient in the error dynamics, as compared to the slow observer gain $L_{2}$ (blue). Moreover, comparing the two sub-figures one sees that the fast allocation is more effective at reducing peaks and transients.

The evolution of the dynamic weights $\Gamma$ is shown in Figure 3 for all the different scenarios. One can notice that the steady-state of the weights is approximately the same for both observer gains $L_{1}$ and $L_{2}$ and for both learning rates $\kappa_{\text {slow }}$ and $\kappa_{\text {fast }}$. The decreasing behavior of the error signal

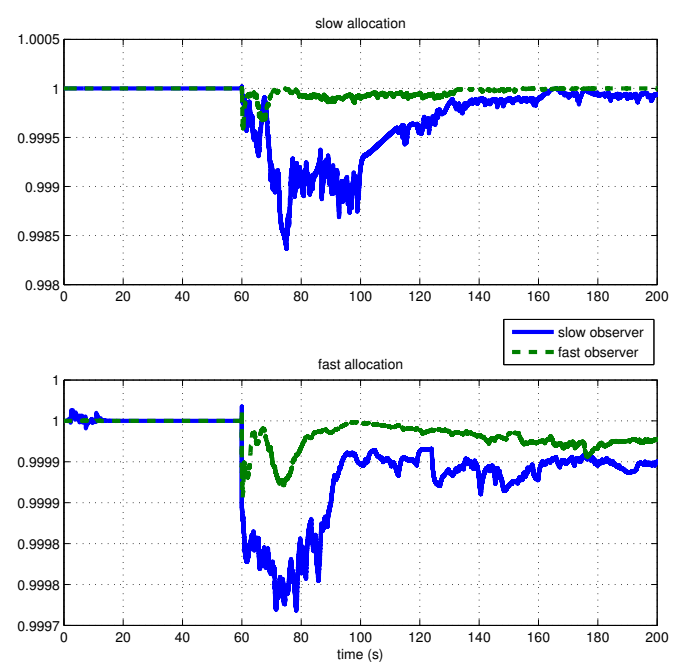

Fig. 5. Example 1: norm of the weights vector $\Gamma$.

$h_{\Gamma}$ after the fault occurrence is depicted in Figure 4, while Figure 5 is a plot of the norm of the vector $\Gamma$.

Example 2: In order to further illustrate the efficiency and the potential capabilities of the proposed dynamic pseudoinverse method, we consider now a second example where the fault affects only one sensor

$$
\phi(t)= \begin{cases}0 & t<85 \\ \phi^{*} & t \geq 85\end{cases}
$$

with $\phi^{*}=\left[\begin{array}{lll}0 & 4 & 0\end{array}\right]^{T}$. The aim of this example is to illustrate that the proposed technique can work well also in the presence of time-varying faults (see the discussion in Remark 5). Moreover, we show that having a timevarying fault may become a key feature to handle one of the critical cases examined in Remark 5, where the matrix $W(\Gamma)$ is rank deficient and hence $W(\Gamma)^{T} W(\Gamma)$ is only positive semidefinite. We consider the same two observer gains in (20) as in the previous example, and a learning rate $\kappa=\kappa_{\text {slow }}=1$. The results are shown in Figures 6-10. We notice that, even though the considered fault is not in the class $\Phi_{0}$, thanks to the persistence of excitation guaranteed by the presence of noise in the outputs, the adaptation law is still working properly and the fault accommodation in the state estimation process is successful (see Figure 6, and Figure 7 for a zoom). As expected, the weight $\gamma_{2}$ converges to zero in order to cancel the effect of the fault: this is clear from the picture in Figure 8. Moreover, as shown respectively in Figure 9 and Figure 10, the norm of the injected signal $h_{\Gamma}$ is rapidly decreasing and the norm of $\Gamma$ is kept essentially constant before and after the fault occurrence.

\section{CONLCUSIONS AND FUTURE WORK}

A new paradigm has been proposed based on a pseudoinverse with adaptive weights when designing linear Luenberger observers in the presence of redundant measurements for linear plants. The proposed scheme is successful at rejecting certain constant faults affecting the sensor measurements as long as certain geometric conditions are satisfied for the fault under consideration. Several simplifying assumptions have been made in this preliminary work, including some 
rank condition on the fault that could be removed in future extensions. We also regard as future work proving global or "in the large" asymptotic stability results, as we only prove local asymptotic stability properties in this note.
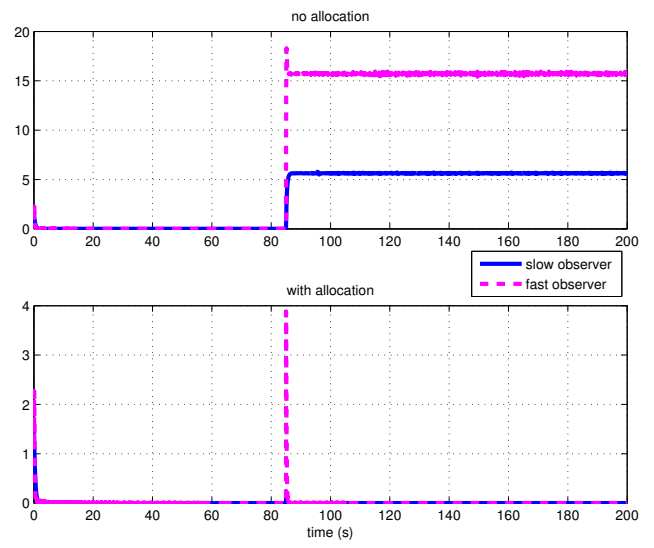

Fig. 6. Example 2: estimation error with and without allocation.

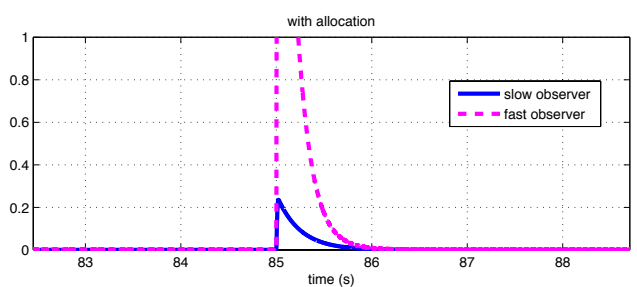

Fig. 7. Example 2: estimation error with allocation (zoom).

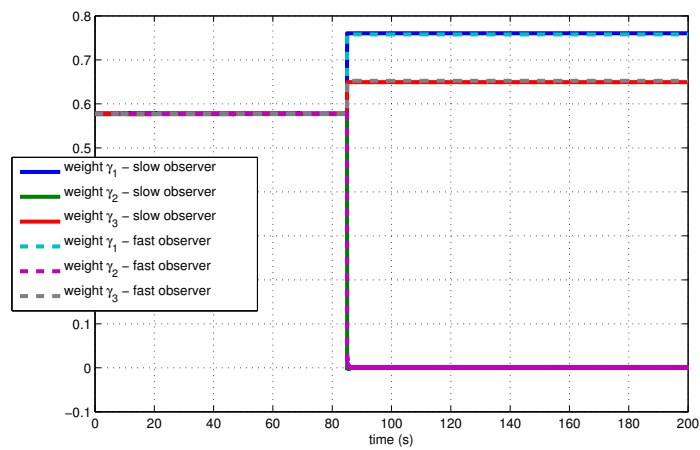

Fig. 8. Example 2: pseudo-inverse weights.

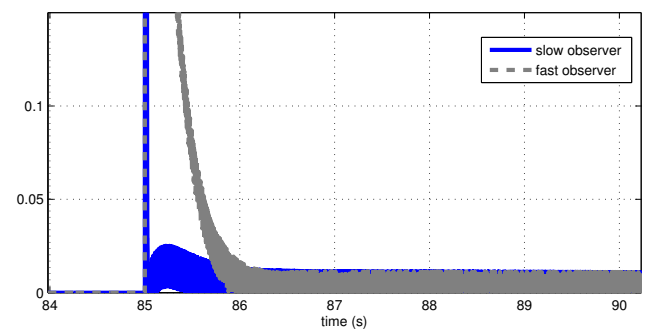

Fig. 9. Example 2: norm of the error signal $h_{\Gamma}$ (zoom).

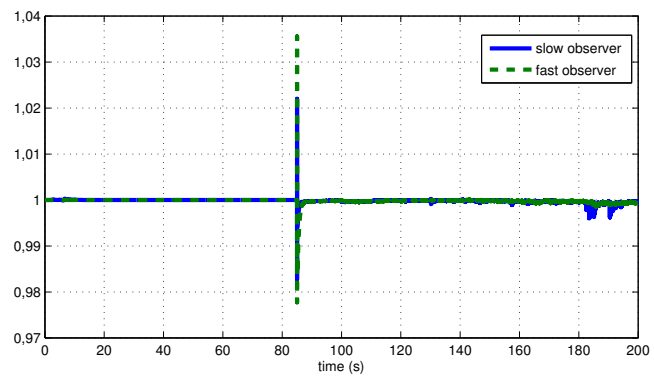

Fig. 10. Example 2: norm of the weights vector $\Gamma$.

\section{REFERENCES}

[1] A. Alessandri and L. Zaccarian. Results on stubborn Luenberger observers for linear time-invariant plants. In European Control Conference (ECC), pages 2920-2925, 2015.

[2] Donald E Catlin. Estimation, control, and the discrete Kalman filter, volume 71. Springer Science \& Business Media, 2012.

[3] Christian Commault, Jean-Michel Dion, Olivier Sename, and Reza Motyeian. Observer-based fault detection and isolation for structured systems. IEEE Transactions on Automatic Control, 47(12):2074-2079, 2002

[4] Dario Corona and Andrea Cristofaro. Some remarks on optima output regulation for weakly dual redundant plants. In 2016 24th Mediterranean Conference on Control and Automation (MED), pages 1205-1211, 2016.

[5] Andrea Cristofaro and Sergio Galeani. Output invisible control allocation with steady-state input optimization for weakly redundant plants. In Proc. 53rd IEEE Conf. Decision and Control, pages 42464253, 2014.

[6] M. Demetriou. Robust adaptive techniques for sensor fault detection and diagnosis. In Proc. 47th IEEE Conf. on Decision and Control, volume 1, pages 1143-1148, 1998.

[7] R. Fitzgerald. Divergence of the Kalman filter. IEEE Trans. on Automatic Control, 16(6):736-747, 1971.

[8] E. Franco, R. Olfati-Saber, T. Parisini, and M. M. Polycarpou. Distributed fault diagnosis using sensor networks and consensus-based filters. In Proc. 45th IEEE Conf. Decision and Control, pages 386391, 2006.

[9] Soren Hansen and Mogens Blanke. Diagnosis of airspeed measurement faults for unmanned aerial vehicles. IEEE Trans. on Aerospace and Electronic Systems, 50(1):224-239, 2014.

[10] T.A. Johansen and T.I. Fossen. Control allocation: A survey. Automatica, page in press, 2013.

[11] Arthur J Krener. Kalman-Bucy and minimax filtering. IEEE Trans, on Automatic Control, 25(2):291-292, 1980.

[12] Rongfu Luo, Manish Misra, S Joe Qin, Randall Barton, and David M Himmelblau. Sensor fault detection via multiscale analysis and nonparametric statistical inference. Industrial \& Engineering Chemistry Research, 37(3):1024-1032, 1998

[13] Amr M Pertew, Horacio J Marquez, and Qing Zhao. LMI-based sensor fault diagnosis for nonlinear lipschitz systems. Automatica, 43(8):1464-1469, 2007.

[14] V. Reppa, M. M. Polycarpou, and C. G. Panayiotou. Adaptive approximation for multiple sensor fault detection and isolation of nonlinear uncertain systems. IEEE Transactions on Neural Networks and Learning Systems, 25(1):137-153, 2014.

[15] Robert H Rogne, Tor A Johansen, and Thor I Fossen. Observer and imu-based detection and isolation of faults in position reference systems and gyrocompasses with dual redundancy in dynamic positioning. In Control Applications (CCA), 2014 IEEE Conference on, pages 83$88,2014$.

[16] Ihab Samy, Ian Postlethwaite, and Da-Wei Gu. Survey and application of sensor fault detection and isolation schemes. Control Engineering Practice, 19(7):658-674, 2011.

[17] L. Zaccarian. Dynamic allocation for input redundant control systems. Automatica, 45:1431-1438, 2009.

[18] Xiaodong Zhang. Sensor bias fault detection and isolation in a class of nonlinear uncertain systems using adaptive estimation. IEEE Trans. on Automatic Control, 56(5):1220-1226, 2011.

[19] Junqiang Zhou, Marcello Canova, and Andrea Serrani. Predictive inverse model allocation for constrained over-actuated linear systems. Automatica, 67:267-276, 2016 